\title{
AXIN2 and SNAIL expression predict the risk of recurrence in cutaneous squamous cell carcinoma after Mohs micrographic surgery
}

\author{
GUOHUA ZHAO $^{1 *}$, KI-YEOL KIM ${ }^{2 *}$, ZHENLONG ZHENG $^{1,3}$, YEONGJOO OH $^{3}$, DAE SAN YOO $^{3}$, \\ MYUNG EUN LEE ${ }^{3}$, KEE YANG CHUNG ${ }^{3}$, MI RYUNG ROH ${ }^{3}$ and ZHEHU JIN ${ }^{1}$ \\ ${ }^{1}$ Department of Dermatology, Yanbian University Hospital, Yanji, Jilin 133000, P.R. China; ${ }^{2}$ Department of Dental Education, \\ BK21 PLUS Project, Yonsei University College of Dentistry, Seoul 03722; ${ }^{3}$ Department of Dermatology, \\ Cutaneous Biology Research Institute, Yonsei University College of Medicine, Seoul 06229, Republic of Korea
}

Received March 20, 2019; Accepted October 17, 2019

DOI: $10.3892 / \mathrm{ol} .2020 .11324$

\begin{abstract}
Recurrence is a common complication observed during cutaneous squamous cell carcinoma (cSCC) treatment; however, biomarkers for predicting recurrence in $\mathrm{cSCC}$ remain unknown. The present study aimed to investigate the predictive value of axis inhibition protein 2 (AXIN2) and SNAIL expression in cSCC recurrence. AXIN2 and SNAIL expression was evaluated using immunohistochemistry in $111 \mathrm{cSCC}$ tissue samples obtained from 18 patients who presented recurrence (recurrence interval, 1-91 months) and 93 patients who did not experience recurrence following Mohs micrographic surgery (MMS) during the follow-up period (156 months). Nomogram construction was performed using patients' clinicopathological characteristics and AXIN2 and SNAIL protein expression. The results demonstrated that high AXIN2 (histoscore >100) and SNAIL (histoscore $>100$ ) expression was detected in 35 and $44 \mathrm{cSCC}$ tissues, respectively. Furthermore, the expression levels of AXIN2 and SNAIL were significantly associated in patients with cSCC $(\mathrm{P}=0.001)$. AXIN2 and SNAIL expression levels were significantly associated with tumor size $(\mathrm{P}=0.021$ and $\mathrm{P}=0.044$, respectively) and recurrence of $\mathrm{cSCC}(\mathrm{P}=0.017$ and $\mathrm{P}=0.042$, respectively). In addition, the results of the
\end{abstract}

Correspondence to: Professor Zhehu Jin, Department of Dermatology, Yanbian University Hospital, 1327 Juzi Road, Yanji, Jilin 133000, P.R. China

E-mail: jinzh_621@163.com

Dr Mi Ryung Roh, Department of Dermatology, Cutaneous Biology Research Institute, Yonsei University College of Medicine, 63 Gil 20 Eonju-ro, Gangnam, Seoul 06229, Republic of Korea

E-mail: karenroh@yuhs.ac

*Contributed equally

Key words: axis inhibition protein 2, SNAIL, predictive nomogram, recurrence, cutaneous squamous cell carcinoma patients, mohs micrographic surgery
Kaplan-Meier curve analysis revealed that recurrence-free survival was significantly associated with tumor size $(\mathrm{P}=0.025)$, differentiation status $(\mathrm{P}<0.001), \mathrm{AXIN} 2$ expression $(\mathrm{P}=0.001)$ and SNAIL expression $(\mathrm{P}=0.001)$. Furthermore, the results of the multivariate analysis demonstrated that age $(\mathrm{P}=0.043)$, AXIN2 expression $(\mathrm{P}=0.001)$ and SNAIL expression $(\mathrm{P}=0.045)$ were independent risk factors for cSCC recurrence in the present cohort. A nomogram for predicting the 1-, 2-, 3-, and 5-year recurrence-free survival was developed for patients with cSCC by including independent risk factors with a concordance index of 0.75 . The results suggested that high AXIN2 and SNAIL expression may be considered as potential risk factors for $\mathrm{cSCC}$ recurrence. This nomogram may therefore be useful to assess the probability of recurrence in patients with cSCC following MMS.

\section{Introduction}

The occurrence of cutaneous squamous cell carcinoma (cSCC), which is the second most common type of non-melanoma skin cancer in Korea, has markedly increased in numerous countries. The age-standardized incidence rate of squamous cell carcinoma during 1999-2014 in Korea was 1.34 per 100,000 people for men, and 1.04 per 100,000 for women. The average annual percentage change (AAPC) of cSCC has increased both in women [AAPC, 6.8 (95\% CI, 5.3 to 8.4)] and men [AAPC, 3.3 (95\% CI, 2.6 to 4.0$)$ ] (1,2). Surgical treatment is curative in most cases of $\mathrm{cSCC}$; in particular, Mohs micrographic surgery (MMS) has become an common treatment option for various types of cutaneous neoplasm, including cSCC (3). As a standard form of tissue-conservative skin cancer surgery, MMS ensures clearance of pathological margins via intraoperative histopathologic interpretation using the fresh-frozen tissue technique; therefore, MMS leads to a lower recurrence rate compared with other therapies that use conventional wide excision (3). However, certain patients that experience recurrence following MMS require adjuvant therapy (3-5). Since adjuvant therapy can cause numerous side effects, it is crucial to identify a reliable method for assessing the risk of recurrence in patients with cSCC following surgery. 
The clinical risk factors for $\mathrm{CSCC}$ recurrence include tumor invasion depth, size, differentiation status, presence of perineural invasion and location (6). In addition, certain molecular biomarkers, including tumor protein 53 , cyclin-dependent kinase inhibitor $2 \mathrm{~A}$, telomerase reverse transcriptase gene (TERT) and programmed cell death ligand 1 (PD-L1) have been considered as potential factors involved in CSCC progression. In particular, TERT promoter mutations and increased PD-L1 expression have been considered as molecular risk factors for cSCC recurrence (7-9). However, these predictive risk factors are inadequate to properly assess the recurrence risk of cSCC with high reproducibility and reliability $(5,6)$.

Epithelial-to-mesenchymal transition (EMT) is a crucial process for cancer cell local invasion and metastasis that acts through the loss of epithelial properties and the acquisition of a mesenchymal phenotype (10). SNAIL, which is a zinc finger transcriptional repressor that functions as a crucial EMT regulator by repressing E-cadherin, is associated with poor prognosis in various types of cancer, such as breast cancer, ovarian cancer, and colorectal cancer (11-13). In cancer cells, activated canonical Wnt signaling inhibits glycogen synthase kinase 3 (GSK-3)-dependent phosphorylation of SNAIL, which subsequently leads to the inhibition of SNAIL degradation, resulting in increased SNAIL protein expression (14). Axis inhibition protein 2 (AXIN2), which is a GSK-3 scaffolding protein and a downstream target of the Wnt signaling pathway, can stabilize nuclear SNAIL expression through the regulation of a nucleocytoplasmic shuttle for GSK-3 (10). Furthermore, it has been reported that AXIN2 expression is positively correlated with SNAIL expression in breast and colon cancer $(10,15)$; however, the predictive value of the expression of these proteins in $\mathrm{CSCC}$ recurrence remains unclear.

This retrospective cohort study aimed to evaluate the predictive value of AXIN2 and SNAIL expression in the recurrence of cSCC and to determine an accurate risk prediction model for $\mathrm{CSCC}$ recurrence.

\section{Materials and methods}

Clinical materials. A total of 111 patients with primary cSCC who had undergone MMS between January 2000 and December 2010 at the Yonsei University Health System, Seoul, Korea, were included in the present study. Patients who had undergone MMS for recurrent $\mathrm{CSCC}$ were excluded from this study. Inclusion criteria were listed as follows: Recurrence of cSCC was first clinically diagnosed and confirmed histologically and histological pattern of cSCC tissue samples were confirmed independently by two pathologists in a blinded manner. All tissue samples were obtained from the Department of Pathology, Yonsei University Health System, Seoul, Korea. The tissue samples were fixed with $4 \%$ formalin, embedded with paraffin, and stored at room temperature (RT) prior to use. This study was approved by the Institutional Review Board for Bioethics of Yonsei University Health System, Severance Hospital (approval no. IRB 4-2018-0331).

IHC. Formalin-fixed paraffin-embedded tissue samples were cut into $4-\mu \mathrm{m}$ tissue sections, deparaffinized with 98.5\% xylene and rehydrated with an ethanol gradient series
(99.9, 80, and 75\% ethanol). Antigen retrieval and blocking of endogenous peroxidase activity were performed using antigen retrieval buffer at $100^{\circ} \mathrm{C}$ for $2 \mathrm{~min}$ (Dako; Agilent Technologies, Inc.) and a mixture of methanol and hydrogen peroxide in a 40:1 ratio at RT for $20 \mathrm{~min}$, respectively. Tissue sections were incubated with the primary antibodies rabbit monoclonal anti-human AXIN2 (Abcam; cat. no. ab109307; 1:250) and polyclonal rabbit anti-human SNAIL (Abcam; cat. no. ab53519; 1:250) at RT for $1 \mathrm{~h}$, as well as REAL EnVision HRP Rabbit/Mouse Detection System at RT for 30 min (Dako; Agilent Technologies, Inc.; cat. no. K5007; prediluted; Agilent Technologies, Inc.) as a secondary antibody, according to the manufacturer's protocols. Visualization and counterstaining were performed using chromogen 3,3'-diaminobenzidine (Dako; Agilent Technologies, Inc.) and hematoxylin at RT for 2 min, respectively. Rabbit IgG (Dako; Agilent Technologies, Inc.) was used as a negative control.

AXIN2 and SNAIL expression levels were evaluated using the weighted histoscore method (16). Briefly, the total histoscore was determined based on tissue staining intensity and percentage of positively stained cells. Staining intensity was scored as $0,1,2$ and 3 for negative, light brown, brown and dark brown staining, respectively. The histoscore was calculated as follows: Total histoscore $=(0 \mathrm{x}$ percentage of negative cells $)+(1 \mathrm{x}$ percentage of light-brown cells $)+(2 \mathrm{x}$ percentage of brown cells $)+(3 \times$ percentage of dark brown cells). For subsequent analysis, patients were subdivided into low and high expression groups with histoscores of 0-100 and 101-300, respectively. The percent of patients with high or low protein expression in each group was calculated and presented in Table I.

Statistical analysis. $\chi^{2}$ and Fisher's exact tests were used to analyze the associations between AXIN2 and SNAIL expression and patients' clinicopathological characteristics. Kaplan-Meier survival curves were plotted based on various clinical factors and protein expression, and the significance was analyzed using the log-rank test to determine the recurrence-free survival of patients with cSCC. Furthermore, the strength of associations among various clinical factors and cSCC recurrence was analyzed using the Cox proportional hazards model. The nomogram for assessment of cSCC recurrence risk was constructed using AXIN2 and Snail expression, as well as various clinicopathological characteristics such as sex, age, lesion site, tumor size, and differentiation, and evaluated with the concordance index (c-index) and a calibration plot (17). R package version 3.1.1 (The R Foundation for Statistical Computing; http://www.r-project.org) with the rms (3.5.0) and eha (2.7.6) packages was used for statistical analysis. $\mathrm{P}<0.05$ was considered to indicate a statistically significant difference.

\section{Results}

Patient clinicopathological characteristics. In the present cohort, $18(16.2 \%)$ patients presented recurrence (interval of recurrence, 1-91 months), whereas 93 (83.8\%) patients did not experience recurrence following MMS during the follow-up period of 156 months. Tissue samples were confirmed independently by two pathologists in a blinded manner. The age 
Table I. Clinicopathological significance of AXIN2 and SNAIL expression in 111 patients with cutaneous squamous cell carcinoma.

\begin{tabular}{|c|c|c|c|c|c|c|c|}
\hline \multirow[b]{2}{*}{ Variable } & \multirow[b]{2}{*}{ Case number, n (\%) } & \multicolumn{3}{|c|}{ AXIN2, n (\%) } & \multicolumn{3}{|c|}{ SNAIL, n (\%) } \\
\hline & & Low & High & $\mathrm{P}$-value & Low & High & P-value \\
\hline \multicolumn{8}{|c|}{ Age, years } \\
\hline$\leq 74$ & $56(50.5)$ & $43(76.8)$ & $13(23.2)$ & \multirow[t]{2}{*}{0.057} & $35(62.5)$ & $21(37.5)$ & \multirow[t]{2}{*}{0.642} \\
\hline$>74$ & $55(49.5)$ & $33(60.0)$ & $22(40.0)$ & & $32(58.2)$ & $23(41.8)$ & \\
\hline \multicolumn{8}{|l|}{$\operatorname{Sex}$} \\
\hline Male & $50(45.0)$ & $35(70.0)$ & $15(30.0)$ & \multirow[t]{2}{*}{0.753} & $28(56.0)$ & $22(44.0)$ & \multirow[t]{2}{*}{0.395} \\
\hline Female & $61(55.0)$ & $41(67.2)$ & $20(32.8)$ & & $39(63.9)$ & $22(36.1)$ & \\
\hline \multicolumn{8}{|l|}{ Site } \\
\hline Scalp & 14 (12.6) & $7(50.0)$ & $7(50.0)$ & \multirow[t]{5}{*}{0.317} & $9(64.3)$ & $5(35.7)$ & \multirow[t]{5}{*}{0.773} \\
\hline Face & 54 (48.6) & $36(66.7)$ & $18(33.3)$ & & $32(59.3)$ & $22(40.7)$ & \\
\hline Ear & $10(9.0)$ & $7(70.0)$ & $3(30.0)$ & & $5(50.0)$ & $5(50.0)$ & \\
\hline Lip & $15(13.5)$ & $13(86.7)$ & $2(13.3)$ & & $11(73.3)$ & $4(26.7)$ & \\
\hline Acral & $18(16.2)$ & $13(72.2)$ & $5(27.8)$ & & $10(55.6)$ & $8(44.4)$ & \\
\hline \multicolumn{8}{|l|}{ Size, $\mathrm{cm}$} \\
\hline$\leq 1.7$ & $56(50.5)$ & $44(78.6)$ & $12(21.4)$ & \multirow[t]{2}{*}{$0.021^{\mathrm{a}}$} & $39(69.6)$ & $17(30.4)$ & \multirow[t]{2}{*}{$0.044^{\mathrm{a}}$} \\
\hline$>1.7$ & $55(49.5)$ & $32(58.2)$ & $23(41.8)$ & & $28(50.9)$ & $27(49.1)$ & \\
\hline \multicolumn{8}{|c|}{ Differentiation } \\
\hline WD & $41(36.9)$ & $27(65.9)$ & $14(34.1)$ & \multirow[t]{3}{*}{0.099} & $25(61.0)$ & $16(39.0)$ & \multirow[t]{3}{*}{0.824} \\
\hline MD & $62(55.9)$ & $46(74.2)$ & $16(25.8)$ & & $38(61.3)$ & $24(38.7)$ & \\
\hline PD & $8(7.2)$ & $3(37.5)$ & $5(62.5)$ & & $4(50.0)$ & $4(50.0)$ & \\
\hline \multicolumn{8}{|c|}{ Recurrence } \\
\hline Yes & $18(16.2)$ & $8(44.4)$ & $10(55.6)$ & \multirow[t]{2}{*}{$0.017^{\mathrm{a}}$} & 7 (38.9) & $11(61.1)$ & \multirow[t]{2}{*}{$0.042^{\mathrm{a}}$} \\
\hline No & $93(83.8)$ & $68(73.1)$ & $25(26.9)$ & & $60(64.5)$ & $33(35.5)$ & \\
\hline
\end{tabular}

${ }^{\mathrm{a}} \mathrm{P}<0.05$. AXIN2, axis inhibition protein 2; MD, moderately differentiated; PD, poorly differentiated; WD, well differentiated.

of patients at diagnosis ranged between 30 and 98 years, with a median age of 74 years. The patient cohort comprised $50(45.0 \%)$ men and $61(55.0 \%)$ women, and the tumor size ranged from $0.3 \mathrm{~cm}$ to $4.5 \mathrm{~cm}$, with a median tumor size of $1.7 \mathrm{~cm}$. cSCC lesions were located on the face $(54 / 111 ; 48.6 \%)$, extremities $(18 / 111 ; 16.2 \%)$, lip $(15 / 111 ; 13.5 \%)$, scalp $(14 / 111$; $12.6 \%)$ and ear $(10 / 111 ; 9.0 \%)$. In addition, the results of the histological grading (18) demonstrated that 41 cases $(36.9 \%)$ were well differentiated (WD), 62 cases $(55.9 \%)$ were moderately differentiated (MD) and 8 cases $(7.2 \%)$ were poorly differentiated (PD). In addition, none of the patients included in this study presented lymph node metastasis or distant metastasis.

AXIN2 and SNAIL expression in cSCC tissue samples. AXIN2, which is a scaffolding protein, was mostly found in the cytoplasm of cancer cells in cSCC tissues. In addition, the results demonstrated that AXIN2 expression was low in $76(68.5 \%)$ and high in $35(31.5 \%)$ cSCC tissues. SNAIL, which is a transcription factor, was found in the cytoplasm and the nucleus of cSCC cells. Furthermore, SNAIL expression was low in $67(60.4 \%)$ and high in $44(39.6 \%)$ cSCC tissues (Table I). Representative expression patterns for AXIN2 and SNAIL IHC are presented in Fig. 1.
A significant association was identified between AXIN2 and SNAIL expression in cSCC tissues. In addition, high SNAIL expression was detected in $62.9 \%$ cSCC tissues with high AXIN2 expression. By contrast, in cSCC tissues with low AXIN2 expression, only $28.9 \%$ exhibited high SNAIL expression (Fig. 2A; $\mathrm{P}=0.001$ ).

Clinicopathological significance of AXIN2 and SNAIL expression in patients with cSCC. High expression levels of AXIN2 and SNAIL were more frequently detected in patients with recurrence (55.6 and $61.1 \%$, respectively) compared with patients without recurrence (26.9 and $35.5 \%$, respectively; $\mathrm{P}=0.017$ and $\mathrm{P}=0.042$, respectively; Fig. 2B and C). Furthermore, tumor size and AXIN2 and SNAIL expression levels were significantly associated in cSCC tissues (Table I). High expression levels of AXIN2 and SNAIL were more frequently detected in patients with larger tumor size (41.8 and 49.1\%, respectively) compared with patients with smaller tumor size (21.4 and $30.4 \%$, respectively; $\mathrm{P}=0.021$ and $\mathrm{P}=0.044$, respectively; Table I). No significant association was identified between AXIN2 and SNAIL protein expression levels and other clinicopathological characteristics, including age, sex, lesion site and differentiation (Table I). 


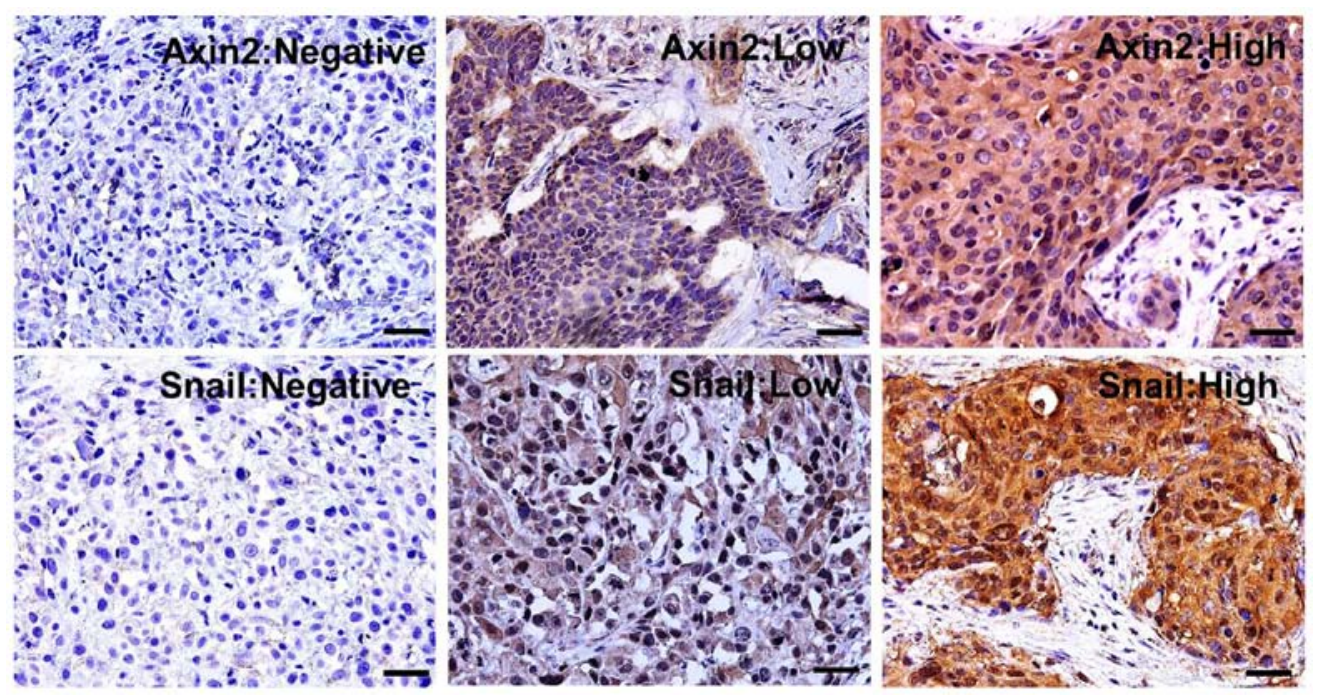

Figure 1. Representative expression patterns of AXIN2 and SNAIL proteins in cutaneous squamous cell carcinoma tissue samples. Magnification, $\mathrm{x} 400$. Scale bar, $20 \mu \mathrm{m}$. Cytoplasmic expression of AXIN2 and cytoplasmic and nuclear expression of SNAIL were considered as positive patterns. AXIN2, axis inhibition protein 2.

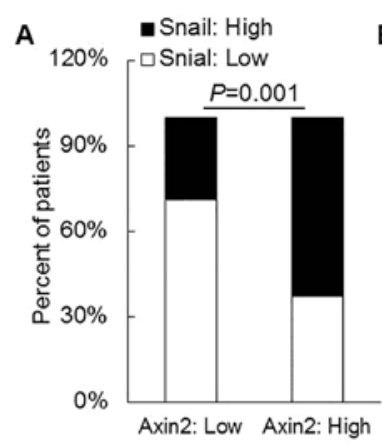

D

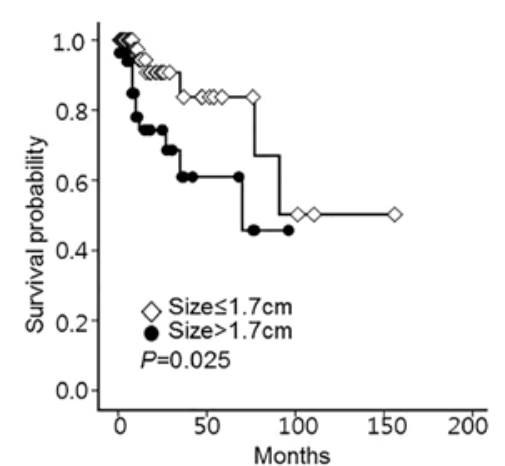

$\mathrm{F}$

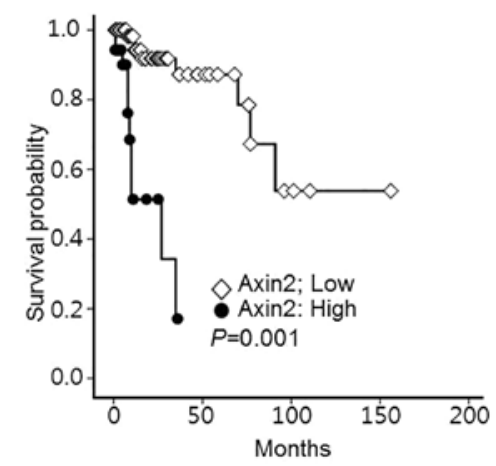

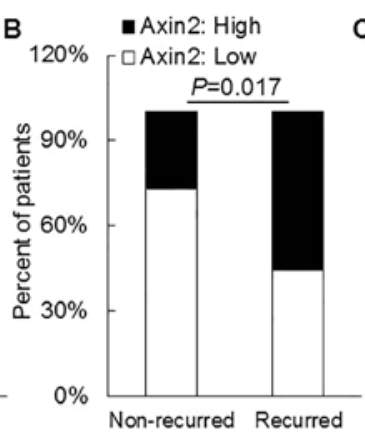

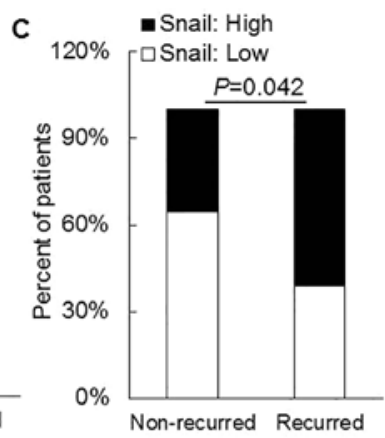

E

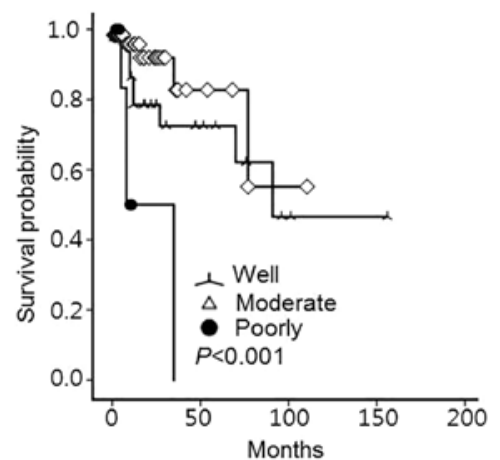

G

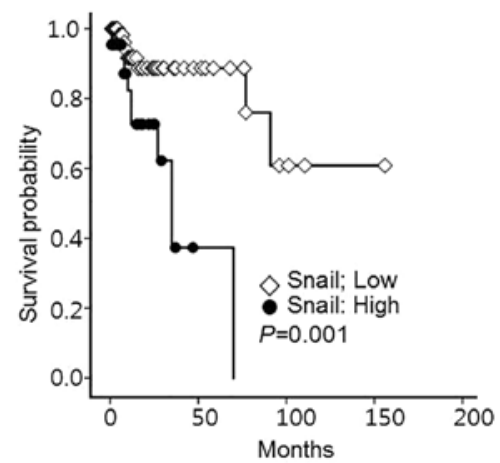

Figure 2. AXIN2 and SNAIL expression in cSCC tissue samples. (A) AXIN2 expression was significantly associated with SNAIL expression in cSCC tissues $\left(\chi^{2}\right.$ test, $\left.\mathrm{P}=0.001\right)$. Recurrence occurred more frequently in patients with (B) high AXIN2 or (C) SNAIL expression compared with patients with low AXIN2 or SNAIL expression (Fisher's exact test, $\mathrm{P}=0.017$ and $\mathrm{P}=0.042$, respectively). Patients with (D) larger tumors, (E) poorly differentiated histological grade, (F) high AXIN2 expression and (G) high SNAIL expression presented decreased recurrence-free survival rates $(\log$-rank test, $\mathrm{P}=0.025, \mathrm{P}<0.001, \mathrm{P}=0.001$ and $\mathrm{P}=0.001$, respectively). AXIN2, axis inhibition protein 2; cSCC, cutaneous squamous cell carcinoma. 
Table II. Risk factors for recurrence in patients with cutaneous squamous cell carcinoma.

\begin{tabular}{lcc}
\hline Variable & Hazard ratio $(95 \% \mathrm{CI})$ & P-value \\
\hline Sex & $1.650(0.492-5.533)$ & 0.417 \\
Age & $0.955(0.914-0.999)$ & $0.043^{\mathrm{a}}$ \\
Lesion site & 1 & \\
Scalp & $0.467(0.104-2.101)$ & 0.321 \\
Face & $0.802(0.103-6.231)$ & 0.833 \\
Ear & $0.409(0.069-2.425)$ & 0.325 \\
Lip & $0.451(0.081-2.541)$ & 0.364 \\
Acral & $1.119(0.297-4.213)$ & 0.868 \\
Size & 1 & \\
Differentiation & $0.817(0.241-2.769)$ & 0.746 \\
WD & $1.516(0.303-7.582)$ & 0.613 \\
MD & $4.795(1.329-17.296)$ & $0.045^{\mathrm{a}}$ \\
PD & $15.169(3.149-73.072)$ & $0.001^{\mathrm{a}}$ \\
SNAIL & & \\
AXIN2 &
\end{tabular}

${ }^{\mathrm{a}} \mathrm{P}<0.05$. CI, Confidence interval; MD, moderately differentiated; $\mathrm{PD}$, poorly differentiated; WD, well differentiated.

Risk factors for recurrence in patients with $c S C C$. The results of the Kaplan-Meier curve analysis demonstrated that recurrence-free survival was significantly associated with tumor size $(\mathrm{P}=0.025)$, differentiation status $(\mathrm{P}<0.001)$ and expression of AXIN2 $(\mathrm{P}=0.001)$ and SNAIL $(\mathrm{P}=0.001$; Fig. 2D-G). In addition, poor recurrence-free survival was observed in patients with tumors of larger size (median survival duration of 13 months for tumor size $\leq 1.7 \mathrm{~cm}$ vs. 8 months for tumor size $>1.7 \mathrm{~cm}$ ), PD histological grade (median survival duration of 12.0 and 11.0 months for WD and MD histological grades, respectively, and of 8.0 months for patients with PD histological grade), high AXIN2 expression (median survival duration of 16.0 months for low AXIN2 expression vs. 6.0 months for high AXIN2 expression) or high SNAIL expression (median survival duration of 13.0 months for low SNAIL expression vs. 8.0 months for high SNAIL expression). According to the American Joint Committee on Cancer (8th edition) (19), tumor diameter $>2 \mathrm{~cm}$ is the main cutoff value for tumor size $(19,20)$. In the present study, no association was identified between tumor size and recurrence-free survival when using the $2 \mathrm{~cm}$ tumor size cut-off value. However, the median tumor size cutoff of $1.7 \mathrm{~cm}$ was more predictive of cumulative disease-free survival rate (Fig. 2D; $\mathrm{P}=0.025$ ). This difference in the cutoff value of tumor size may be due to the difference between MMS and other surgical methods.

The results of the Cox-regression analysis demonstrated that age [hazard ratio (HR), 0.955; 95\% confidence interval (CI), 0.914-0.999; $\mathrm{P}=0.043$ ], high $\mathrm{AXIN} 2$ expression (HR, 15.169; 95\% CI, 3.149-73.072; $\mathrm{P}=0.001)$ and high SNAIL expression (HR, 4.795; 95\% CI, 1.329-17.296; $\mathrm{P}=0.045$ ) were independent risk factors for recurrence-free survival in patients with cSCC (Table II).
Construction of a predictive nomogram. Predictive nomograms were constructed using patients' clinicopathological characteristics and/or AXIN2 and SNAIL protein expression to analyze the 1-, 2-, 3- and 5-year recurrence-free survival in patients with cSCC. The c-index of the nomogram constructed with a combination of clinicopathological characteristics, including sex, age, lesion site, tumor size and differentiation, was $\sim 0.40$. When the nomogram was constructed using AXIN2 and SNAIL protein expression, the c-index was $\sim 0.69$. When all clinicopathological characteristics and AXIN2 and SNAIL protein expression were combined, the c-index of the nomogram was $\sim 0.61$. Furthermore, the c-index increased to $\sim 0.75$ when the nomogram was constructed with independent risk factors such as age and expression of AXIN2 and SNAIL (Fig. 3).

To demonstrate the practical usage of the nomogram, a nomogram in Hypertext Markup Language (HTML) format that included predictive factors, total score and probabilities of recurrence-free survival was constructed (Fig. 4A). As presented in Fig. 4Aa, in a patient aged 54 years with low AXIN2 and high SNAIL expression, the probability of recurrence-free survival calculated using the nomogram was 99, 92.5, 84.6 and $77.6 \%$ after 1, 2, 3 and 5 years, respectively. By contrast, as presented in Fig. 4Ab, a 54-year-old patient with high AXIN2 expression and low SNAIL expression presented a probability of recurrence-free survival of 82.8, 66.2, 54.3 and $35.5 \%$ after 1, 2, 3 and 5 years, respectively. The constructed nomogram with the highest c-index was calibrated to further evaluate the association between the predicted probability and the real outcomes of patients with cSCC. The predicted outcomes are presented on the $\mathrm{x}$-axis, and the actual data for recurrence-free survival of each patient with cSCC are presented on the y-axis. An ideal nomogram would demonstrate perfect consensus $(\mathrm{x}=\mathrm{y})$ between data predicted by the nomogram and actual recurrence-free survival for each patient in the cohort. The results of the present study demonstrated a relatively solid line, especially for the prediction of recurrence-free survival after 1 and 2 years (Fig. 4B).

\section{Discussion}

Aberrant activation of the Wnt signaling pathway and subsequent initiation of EMT have been demonstrated to be associated with poor prognosis in various types of cancer, including cSCC $(10,14,21)$. In the present study, the predictive value of the protein expression of two EMT genes, AXIN2 and SNAIL, in the recurrence of $\mathrm{CSCC}$ was evaluated over a long-term follow-up period.

Wnt signaling is activated by binding of the Wnt ligand to its co-receptors, including seven-transmembrane-domain Frizzled receptors and a low-density lipoprotein (LDL) receptor-related protein (LRP5/LRP6). In the absence of Wnt ligands, GSK 3 assembles a $\beta$-catenin destruction complex and subsequently mediates $\beta$-catenin degradation (22). Previous studies reported that AXIN2 is a molecular component of the $\beta$-catenin destruction complex that can repress various downstream target genes of $\beta$-catenin, indicating its role as a tumor suppressor. However, when aberrant activation of the Wnt signaling pathway occurs, AXIN2 protein is translocated from the $\beta$-catenin destruction complex to the LRP5 receptor via the phosphorylated Dishevelled, leading to the inactivation of 
Points

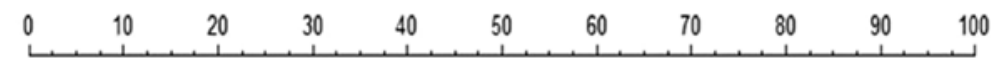

Age

Axin2

Snail

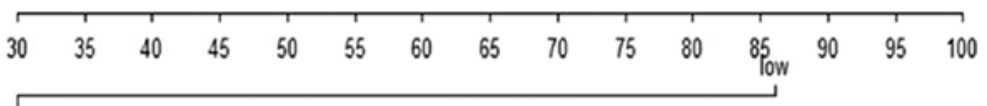

Total points

Linear predictor

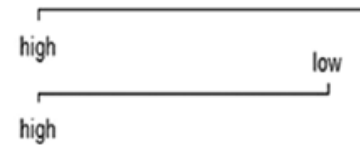

high

Probability of 1-year RFS

$2 \begin{array}{lllllllllll}1 & 2.5 & 3 & 3.5 & 4 & 4.5 & 5 & 5.5 & 6 & 6.5 & 7\end{array}$

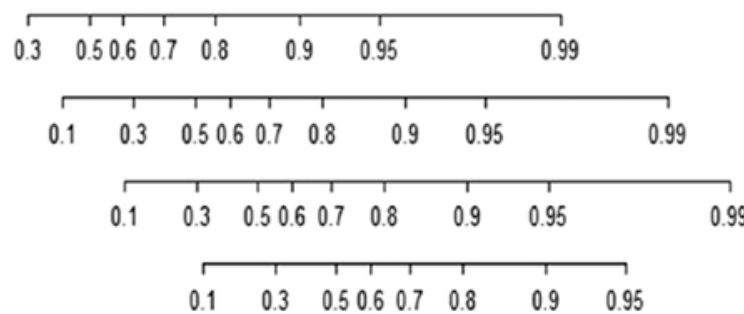

Probability of 2-year RFS

Probability of 3-year RFS

Probability of 5-year RFS

Figure 3. Nomogram for predicting the probability of RFS of cSCC patients. Probability of 1-, 2-, 3- and 5-year RFS for each patient with cSCC may be identified using the nomogram. AXIN2, axis inhibition protein 2; $\mathrm{CSCC}$, cutaneous squamous cell carcinoma; RFS, recurrence-free survival.

A

(a)

Please check the status of a patient

Age

\begin{tabular}{l}
\hline 54 \\
\hline Axin2 \\
○ Low \\
Snail \\
O Low High
\end{tabular}

\begin{tabular}{|l|l|l|}
\hline Total Score & \multicolumn{1}{|c|}{156} \\
\hline Probability of 1-year RFS & 0.99 \\
\hline Probability of 2-year RFS & 0.9249909023 \\
\hline Probability of 3-year RFS & 0.8463126189 \\
\hline Probability of 5-year RFS & 0.7760472522 \\
\hline
\end{tabular}

B

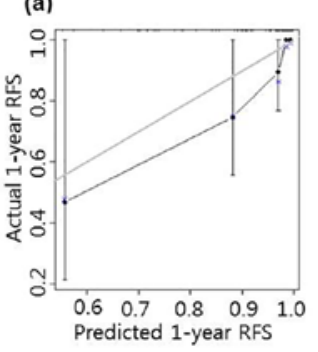

(b)

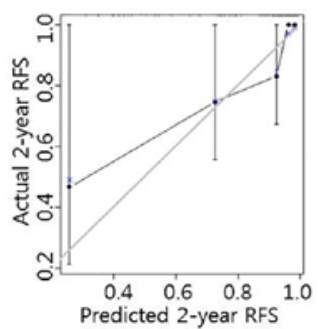

(b)

Please check the status of a patient
Age
\begin{tabular}{|l|}
\hline 54 \\
Axin2 \\
OLow $\odot$ High \\
Snail \\
○ Low O High
\end{tabular}

\begin{tabular}{|l|l|}
\hline Total Score & \multicolumn{1}{|c|}{107} \\
\hline Probability of 1-year RFS & 0.8275468803 \\
\hline Probability of 2-year RFS & 0.6615884086 \\
\hline Probability of 3-year RFS & 0.5428924611 \\
\hline Probability of 5-year RFS & 0.3551491976 \\
\hline
\end{tabular}

(c)

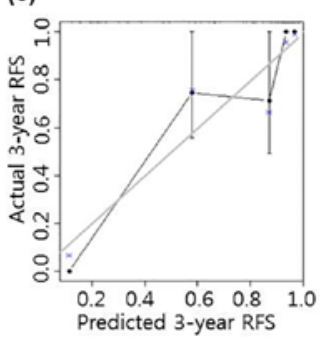

(d)

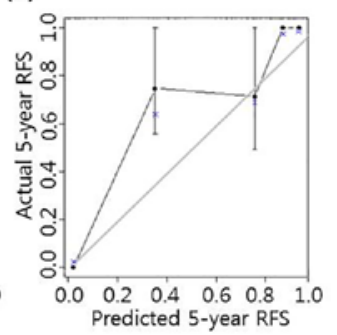

Figure 4. (A-a and A-b) HTML format for the nomogram presenting highest accuracy. HTML showed the total score and the associated 1-, 2-, 3- and 5-year RFS for patients with cSCC. (Ba-Bd) Calibration of the nomogram. An ideal nomogram would demonstrate perfect consensus ( $\mathrm{x}=\mathrm{y}$ ) between data predicted by the nomogram and actual RFS for each patient in the cohort. The calibration of the nomogram demonstrated a relatively solid line, especially for the prediction of RFS after 1 and 2 years. AXIN2, axis inhibition protein 2; cSCC, cutaneous squamous cell carcinoma; HTML, Hypertext Markup Language; RFS, recurrence-free survival.

the $\beta$-catenin destruction complex. $\beta$-catenin is subsequently protected from the degradation process and further involved in the transcription of Wnt target genes $(23,24)$.
Previous studies suggested that AXIN2 may serve oncogenic roles. As one of the T-cell factor/lymphoid enhancer factor downstream targets of canonical Wnt signaling 
pathway, AXIN2 has been demonstrated to be upregulated in various types of cancer, including hepatoblastoma, cervical, breast and colorectal cancer $(10,25,26)$. Furthermore, a study has reported that AXIN2 upregulation mediates the increased nuclear SNAIL and $\beta$-catenin expression by supporting the nuclear export function of GSK-3 in breast cancer cells (10). In addition, the present study demonstrated that AXIN2 and SNAIL expression levels were significantly associated in cSCC tissues. A previous study reported that AXIN2 knockdown led to decreased invasive ability of colorectal cancer cells (15), whereas another study demonstrated that AXIN2 can induce genomic instability by influencing centrosome cohesion at the mitotic checkpoint (27). These findings suggested that AXIN2 may act as an oncogene protein during cancer progression, but not as a tumor suppressor.

Consistent with observations in breast cancer (10), the results of the present study reported that SNAIL expression was more frequently detected in $\mathrm{cSCC}$ tissues with high AXIN2 expression compared with cSCC tissues with low AXIN2 expression. Furthermore, the results of the Cox regression analysis demonstrated that the expression of AXIN2 and SNAIL was an independent risk factor for recurrence-free survival in patients with cSCC. In particular, patients with high AXIN2 and SNAIL expression presented a higher risk of recurrence compared with patients with low AXIN2 and SNAIL expression or patients with high expression of AXIN2 or SNAIL only. These results suggested that AXIN2-mediated SNAIL stabilization and aberrant activation of the Wnt signaling pathway may serve crucial roles in cSCC recurrence.

In the present study, certain clinicopathological characteristics, including large tumor size and poor tumor differentiation, were also identified as risk factors for recurrence in patients with cSCC according to the Kaplan-Meier analysis. Furthermore, age was considered as an independent risk factor for recurrence in this study. However, when the nomogram was constructed using only clinicopathological characteristics, the prediction model accuracy was very low, the c-index of the nomogram was $\sim 0.40$. The accuracy was improved by considering the molecular markers as predictive factors. The c-index increased to $\sim 0.75$ when the clinicopathological characteristics were combined with AXIN2 and SNAIL expression. In addition, the results of the calibration plot also demonstrated that the nomogram exhibited a high level of predictive accuracy in patients with cSCC. Further identification of novel biomarkers related to $\mathrm{CSCC}$ pathogenesis are required in order to increase the prediction accuracy of the nomogram.

Since AXIN2 is a target of the canonical Wnt signaling pathway, the results of the present study suggested that activation of the canonical Wnt signaling pathway and EMT progression may be involved in cSCC recurrence. In addition, AXIN2 and SNAIL expression may be considered as potential predictive biomarkers for assessing the risk of recurrence in patients with cSCC.

The present study had limitations. Certain important clinical characteristics, including previous history of organ transplantation, diabetes mellitus and other cancers, were excluded from analysis as they were missing in $>50 \%$ patients. The predictive nomogram constructed in the present study may therefore not be fully performant. However, to the best of our knowledge, the present study described the first prediction model that may be used to investigate the risk of recurrence in patients with cSCC following MMS.

\section{Acknowledgements}

The authors would like to thank Professor Haiyue Chen and Dr Meici Piao for histological evaluation.

\section{Funding}

This study was supported by the National Natural Science Foundation of China (grant no. 81560503) and the National Research Foundation of Korea (grant no. 2017R1C1B2005574 and 2019R1A2C1003028).

\section{Availability of data and materials}

The datasets used and/or analyzed during the present study are available from the corresponding author on reasonable request.

\section{Authors' contributions}

Histological analysis was performed by GZ and ZZ. GZ, ZZ, and YO performed immunohistochemistry for AXIN2 and SNAIL. KK, ML and DY analyzed the data. KC, MR and ZJ conceived the study and wrote the manuscript. All authors read and approved the final version of the manuscript.

\section{Ethics approval and consent to participate}

This study was approved by the Institutional Review Board for Bioethics of Yonsei University Health System, Severance Hospital (approval no. IRB 2018-0874-001). Patient consent for using the stored tissue was exempted by approval of IRB.

\section{Patient consent for publication}

Not applicable.

\section{Competing interests}

The authors declare that they have no competing interests.

\section{References}

1. Oh CM, Cho H, Won YJ, Kong HJ, Roh YH, Jeong KH and Jung KW: Nationwide trends in the incidence of melanoma and non-melanoma skin cancers from 1999 to 2014 in South Korea. Cancer Res Treat 50: 729-737, 2018.

2. Lobeck A, Weiss C, Orouji A, Koch PS, Heck M, Utikal J, Koenen W, Faulhaber J, Klemke CD and Felcht M: Single center analysis of the dermatosurgical patient cohort of a tumor center in Germany. Hautarzt 68: 377-384, 2017 (In German).

3. Le ST, Kamal HY and Khachemoune A: Mohs micrographic surgery for cutaneous malignancies: A focus review of its indications in pediatric age groups. Pediatr Dermatol 35: 434-440, 2018.

4. Schmults CD, Karia PS, Carter JB, Han J and Qureshi AA: Factors predictive of recurrence and death from cutaneous squamous cell carcinoma: A 10-year, single-institution cohort study. JAMA Dermatol 149: 541-547, 2013.

5. Thompson AK, Kelley BF, Prokop LJ, Murad MH and Baum CL: Risk factors for cutaneous squamous cell carcinoma recurrence, metastasis, and disease-specific death: A systematic review and meta-analysis. JAMA Dermatol 152: 419-428, 2016. 
6. Roscher I, Falk RS, Vos L, Clausen OPF, Helsing P, Gjersvik P and Robsahm TE: Validating 4 staging systems for cutaneous squamous cell carcinoma using population-based data: A nested case-control study. JAMA Dermatol 154: 428-434, 2018.

7. Que SKT, Zwald FO and Schmults CD: Cutaneous squamous cell carcinoma: Incidence, risk factors, diagnosis, and staging. J Am Acad Dermatol 78: 237-247, 2018.

8. Campos MA, Macedo S, Fernandes M, Pestana A, Pardal J, Batista R, Vinagre J, Sanches A, Baptista A, Lopes JM and Soares P: TERT promoter mutations are associated with poor prognosis in cutaneous squamous cell carcinoma. J Am Acad Dermatol 80: 660-669.e6, 2019.

9. Garcia-Diez I, Hernandez-Ruiz E, Andrades E, Gimeno J, Ferrándiz-Pulido C, Yébenes M, García-Patos V, Pujol RM, Hernández-Muñoz I and Toll A: PD-L1 expression is increased in metastasizing squamous cell carcinomas and their metastases. Am J Dermatopathol 40: 647-654, 2018.

10. Yook JI, Li XY, Ota I, Hu C, Kim HS, Kim NH, Cha SY, Ryu JK, Choi YJ, Kim J, et al: A Wnt-Axin2-GSK3beta cascade regulates Snaill activity in breast cancer cells. Nat Cell Biol 8: 1398-1406, 2006.

11. Muenst S, Daster S, Obermann EC, Droeser RA, Weber WP, von Holzen U, Gao F, Viehl C, Oertli D and Soysal SD: Nuclear expression of snail is an independent negative prognostic factor in human breast cancer. Dis Markers 35: 337-344, 2013.

12. Wang YL, Zhao XM, Shuai ZF, Li CY, Bai QY, Yu XW and Wen QT: Snail promotes epithelial-mesenchymal transition and invasiveness in human ovarian cancer cells. Int J Clin Exp Med 8: 7388-7393, 2015.

13. Brzozowa M, Michalski M, Wyrobiec G, Piecuch A, Dittfeld A, Harabin-Słowińska M, Boroń D and Wojnicz R: The role of Snaill transcription factor in colorectal cancer progression and metastasis. Contemp Oncol (Pozn) 19: 265-270, 2015.

14. Yook JI, Li XY, Ota I, Fearon ER and Weiss SJ: Wnt-dependent regulation of the E-cadherin repressor snail. J Biol Chem 280: 11740-11748, 2005.

15. Wu ZQ, Brabletz T, Fearon E, Willis AL, Hu CY, Li XY and Weiss SJ: Canonical Wnt suppressor, Axin2, promotes colon carcinoma oncogenic activity. Proc Natl Acad Sci USA 109: 11312-11317, 2012

16. Zhang X, Zheng Z, Shin YK, Kim KY, Rha SY, Noh SH, Chung HC and Jeung HC: Angiogenic factor thymidine phosphorylase associates with angiogenesis and lymphangiogenesis in the intestinal-type gastric cancer. Pathology 46: 316-324, 2014
17. Chen F, Lin L, Yan L, Liu F, Qiu Y, Wang J, Hu Z, Wu J, Bao X, Lin L, et al: Nomograms and risk scores for predicting the risk of oral cancer in different sexes: A large-scale case-control study. J Cancer 9: 2543-2548, 2018.

18. Burton KA, Ashack KA and Khachemoune A: Cutaneous squamous cell carcinoma: A review of high-risk and metastatic disease. Am J Clin Dermatol 17: 491-508, 2016.

19. Amin MB, Edge S, Greene F, Byrd DR, Brookland RK, Washington MK, Gershenwald JE, Compton CC, Hess KR, Sullivan DC, et al (eds): AJCC Cancer Staging Manual (8th edition). New York, Springer, 2017.

20. Mullen JT, Feng L, Xing Y, Mansfield PF, Gershenwald JE, Lee JE, Ross MI and Cormier JN: Invasive squamous cell carcinoma of the skin: Defining a high-risk group. Ann Surg Oncol 13: 902-909, 2006

21. Halifu Y, Liang JQ, Zeng XW, Ding Y, Zhang XY, Jin TB, Yakeya B, Abudu D, Zhou YM, Liu XM, et al: Wnt1 and SFRP1 as potential prognostic factors and therapeutic targets in cutaneous squamous cell carcinoma. Genet Mol Res 15, 2016.

22. Taketo MM: Shutting down Wnt signal-activated cancer. Nat Genet 36: 320-322, 2004.

23. Mao J, Wang J, Liu B, Pan W, Farr GH III, Flynn C, Yuan H, Takada S, Kimelman D, Li L and Wu D: Low-density lipoprotein receptor-related protein-5 binds to Axin and regulates the canonical Wnt signaling pathway. Mol Cell 7: 801-809, 2001.

24. Cadigan KM and Liu YI: Wnt signaling: Complexity at the surface. J Cell Sci 119: 395-402, 2006.

25. Hughes TA and Brady HJ: Regulation of axin2 expression at the levels of transcription, translation and protein stability in lung and colon cancer. Cancer Lett 233: 338-347, 2006.

26. Lustig B, Jerchow B, Sachs M, Weiler S, Pietsch T, Karsten U, van de Wetering M, Clevers H, Schlag PM, Birchmeier W and Behrens J: Negative feedback loop of Wnt signaling through upregulation of conductin/axin2 in colorectal and liver tumors. Mol Cell Biol 22: 1184-1193, 2002.

27. Hadjihannas MV, Bruckner $\mathrm{M}$ and Behrens J: Conductin/axin2 and Wnt signalling regulates centrosome cohesion. EMBO Rep 11: 317-324, 2010 .

This work is licensed under a Creative Commons Attribution-NonCommercial-NoDerivatives 4.0 International (CC BY-NC-ND 4.0) License. 